\title{
Protótipo do aplicativo Ergon para informação e conscientização ergonômica
}

\author{
Adakrishna Sampaio Saraiva Bitencourte', Márcia Maria Pereira Rendeiro² \\ ${ }^{1}$ Mestranda do Mestrado Profissional em Telemedicina e Telessaúde - Universidade do \\ Estado do Rio de Janeiro (UERJ) - Rio de Janeiro - RJ - Brazil \\ ${ }^{2}$ Coordenadora Adjunta do Mestrado Profissional em Telemedicina e Telessaúde. \\ Universidade do Estado do Rio de Janeiro (UERJ) - Rio de Janeiro - RJ - Brazil \\ adaergon@gmail.com, mmrendeiro@yahoo.com
}

\begin{abstract}
This work shows a prototype mobile aplication (app), called Ergon, which allows access to information for ergonomic awareness of companies and workers, to contribute in identification and prevention actions for ergonomic risks that can results in damage to workers' health. Apps' content was based on the work aspects conditions covered in Ergonomics Standard 17 - NR17, of Brazil Labour Secretary.
\end{abstract}

Resumo. Este trabalho apresenta o protótipo do aplicativo (app) Ergon, o qual permite o acesso às informações para conscientização ergonômica de empresas e trabalhadores, com objetivo de contribuir nas ações de identificação e prevenção de riscos ergonômicos que podem acarretar em danos à saúde do trabalhador. O conteúdo do app foi baseado nos aspectos das condições de trabalho abordadas na Norma Regulamentadora 17 - NR 17, da Secretaria do Trabalho do Brasil.

\section{Introdução}

A Ergonomia é o estudo da adaptação do trabalho ao ser humano, abrangendo os trabalhos executados com máquinas e equipamentos e todas as situações em qual ocorre relacionamento entre o ser humano e uma atividade produtiva de bens ou serviços [Iida, 2016]. Esta disciplina busca entender as interações entre os seres humanos e outros elementos ou sistemas, aplicando teorias, princípios, dados e métodos a projetos a fim de otimizar o bem estar humano e o desempenho global do Sistema [ABERGO, 2008]. O campo de Saúde do Trabalhador pode utilizar a Ergonomia como um instrumento de análise dos riscos ergonômicos presentes em uma situação de trabalho com objetivo de prevenção de lesões e doenças.

Segundo dados preliminares do Instituto Nacional de Seguridade Social (INSS), em 2017 foram concedidos 22.029 benefícios acidentários a trabalhadores que precisaram ficar mais de 15 dias afastados do trabalho por causa de algum tipo de doença relacionada à Lesão por Esforço Repetitivo e Distúrbio Osteomuscular Relacionado ao Trabalho - LER/DORT. O número representa $11,19 \%$ de todos os benefícios concedidos. Essas lesões podem ocorrer devido a tarefas repetitivas que exigem força ou um ritmo de trabalho acelerado, somadas a posturas inadequadas e ao estresse, formando um ambiente propício para o surgimento dessas lesões ou doenças. [BRASIL, 2018]. 
A prevenção às lesões e doenças relacionadas ao trabalho é uma temática explorada na Secretaria do Trabalho (ST), através das normas regulamentadoras (NRs), que possuem caráter obrigatório para empresas públicas e privadas, e fornecem os parâmetros para adequação em Saúde e Segurança no Trabalho, no caso da Ergonomia, a Norma Regulamentadora 17 - NR 17. Os treinamentos fazem parte dos meios para identificação e mitigação dos riscos ergonômicos, possibilitando a melhoria da condição de trabalho pela empresa e trabalhadores em situações que não necessitem de um profissional especializado.

Existe um impacto social e econômico no movimento atual de migração das empresas das capitais para cidades do interior devido aos maiores incentivos fiscais, menores custos de produção - em casos de utilização de matéria-prima originária do interior ou contratação de mão de obra mais barata, menor índice de violência e melhores condições de logística [JUNIOR, 2016]. Essa migração pode dificultar o acesso ao ergonomista, trazendo a necessidade de autonomia das empresas e trabalhadores, nas situações simples de obtenção de um ambiente de trabalho seguro e saudável.

A ergonomia de conscientização procura capacitar os próprios trabalhadores para a identificação e correção dos problemas do dia a dia ou aqueles emergenciais, pois problemas ergonômicos podem não ser solucionados na fase de concepção ou na fase de correção. A conscientização geralmente é feita por meio de cursos de treinamento e reciclagens, ensinando o trabalhador a operar de forma segura, reconhecendo os fatores de risco que podem surgir a qualquer momento no ambiente de trabalho [Iida, 2016].

As Tecnologias da Informação e Comunicações (TICs) são utilizadas na capacitação a distância e, atualmente, têm nos equipamentos móveis um destaque por sua sofisticação técnica e crescente presença na vida das pessoas e das organizações, trazendo a flexibilização da execução das tarefas no local e tempo de sua preferência ou possibilidade [OLIVEIRA et al, 2015]. Com a característica de ubiquidade dos equipamentos móveis, o presente estudo sugere que estes sejam utilizados como uma ferramenta para a conscientização ergonômica em empresas, possibilitando ao usuário o seu acesso a qualquer hora e local.

Foi realizado um estudo preliminar ao desenvolvimento do aplicativo Ergon que demonstrou uma carência de aplicativos informativos ou de conscientização ergonômica, disponíveis nas lojas virtuais. Somente um aplicativo, denominado Pontos de Verificação Ergonômica, mais se aproximou das condições de trabalho e itens da NR 17.

O presente estudo visa demonstrar o protótipo do aplicativo móvel Ergon, que tem o propósito de facilitar o acesso à informação e conscientização ergonômica, baseado na NR 17, para a prevenção de LER/DORT.

\section{Etapas do desenvolvimento do App}

Para a concepção do app Ergon foram programadas cinco etapas de desenvolvimento:

Etapa 1. Análise dos aplicativos informativos em ergonomia, definição do conteúdo e objetivo do aplicativo: nesta fase foi realizada uma revisão de literatura e um estudo dos 
aplicativos informativos de Ergonomia disponíveis nas lojas virtuais Google Play e Play Store. Posteriormente, foi elaborado o conteúdo para conscientização ergonômica.

Etapa 2. Planejamento das interfaces e da navegabilidade: nesta fase foi projetado o lay out estático do aplicativo junto à um designer com uso do programa gráfico Corel Draw.

Etapa 3. Desenvolvimento do protótipo: esta fase está prevista a ser executada junto à uma agência de publicidade para execução do wireframe do aplicativo com possibilidade de criação de aplicações para as plataformas Android e iOS.

Etapa 4. Teste de validação do protótipo: serão realizados testes com usuários na área de Ergonomia, lideranças de áreas produtivas de empresas, trabalhadores e profissionais e informática, com avaliação do aplicativo por uso de questionário no google forms.

Etapa 5: Ajustes e refinamento do protótipo. Os problemas identificados na fase de validação serão ajustados para a concepção do produto final.

\section{Protótipo do app Ergon: fluxo de navegação e interfaces}

A construção do conteúdo do aplicativo se fez por conceituações ergonômicas para identificação de riscos e implementação de melhorias baseadas nos temas abordados pela NR 17. Os temas abordados no aplicativo foram: conceitos de ergonomia, riscos ergonômicos, informações e sugestões de melhorias para as cinco condições de trabalho abordadas sobre a NR 17 - levantamento, transporte e descarga de materiais, mobiliário, equipamentos, condições ambientais do posto de trabalho e organização do trabalho. Para isso foram elaboradas 38 telas com textos e pictogramas, buscando fácil entendimento para trabalhadores em geral. A figura 1 ilustra o fluxo de navegação e a organização das informações e orientações do protótipo do aplicativo:

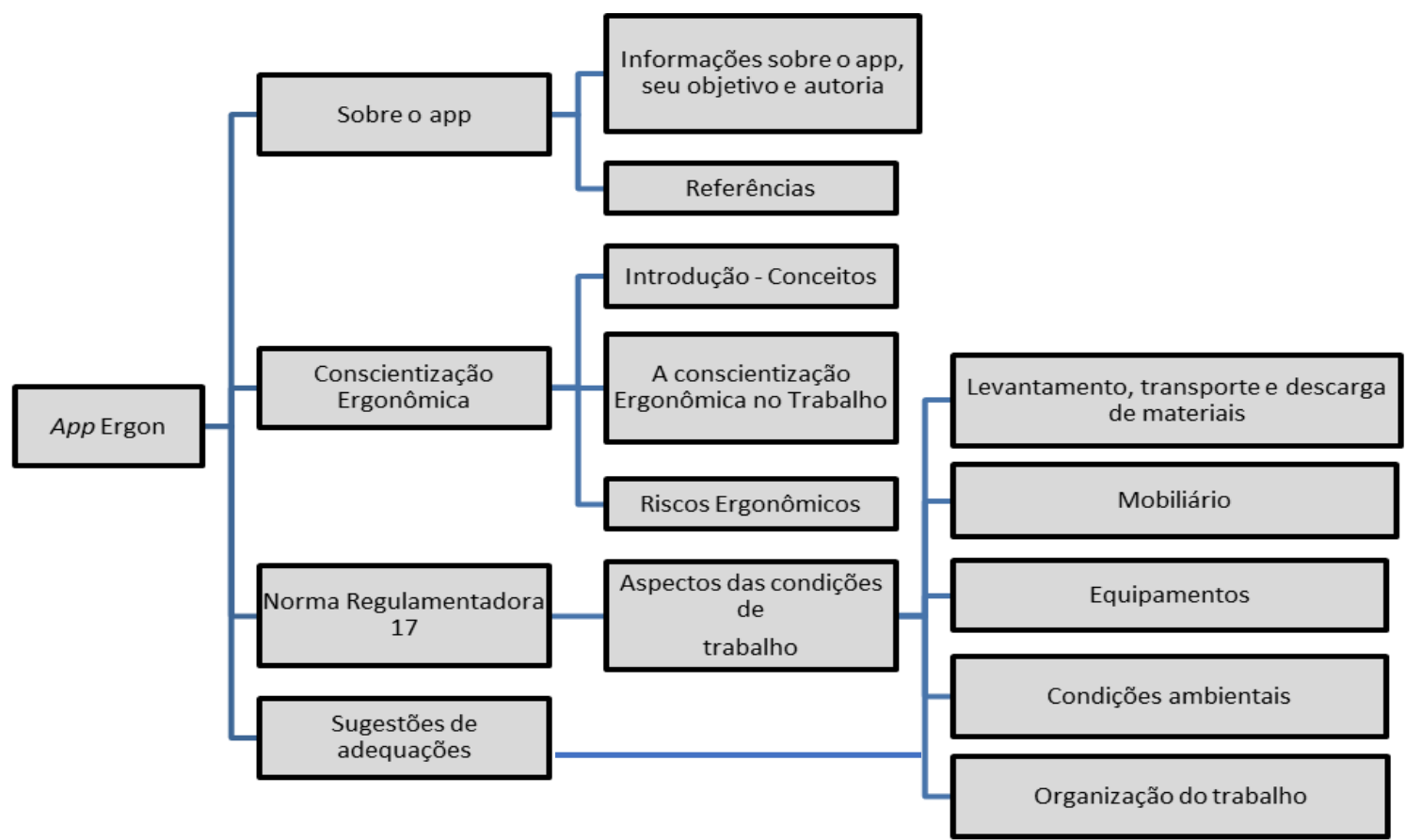

Figura 1. Fluxo de navegação e organização das informações do aplicativo Ergon. Fonte: Autores. 
A interface do aplicativo foi dividida em quatro partes, que são as opções de informações principais e iniciais para navegação: a explicação sobre o aplicativo, a conscientização ergonômica com conceitos e descrição de riscos ergonômicos, a legislação vigente de ergonomia - NR 17 e seus aspectos abordados, por fim as sugestões de adequações.

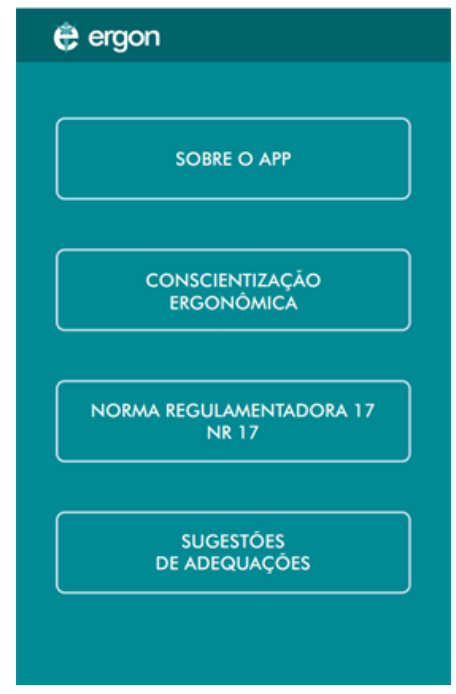

Figura 2. Tela das opções de informações principais do aplicativo

Fonte: Autores.
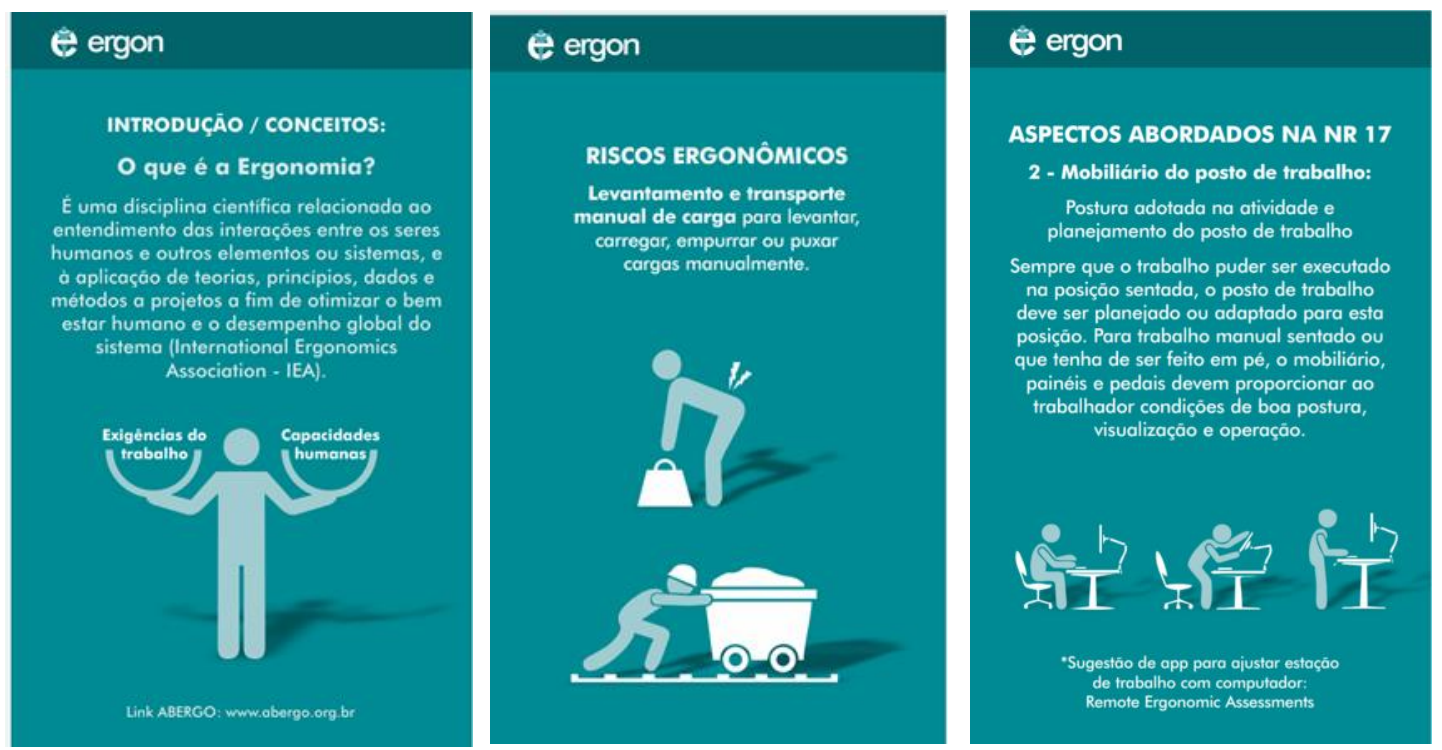

Figura 3. Tela com informações para conscientização ergonômica.

Fonte: Autores.

É importante ressaltar que o aplicativo Ergon não substitui a Análise Ergonômica do Trabalho realizada por Ergonomista. 


\section{Discussão}

Godderis e Lambrechts [2019] consideram o uso das novas tecnologias de saúde eletrônica como uma oportunidade para a saúde e segurança no trabalho. Para eles o local de trabalho desempenha um papel essencial na promoção de um estilo de vida saudável, devido ao tempo que muitos funcionários passam no trabalho.

A Organização Internacional do Trabalho (OIT) junto à International Ergonomics Association (IEA) desenvolveram o manual Ergonomic Chekpoints, já traduzido para o português como Pontos de Verificação Ergonômica, com o intuito de preencher alguns vazios, particularmente para pequenas e médias empresas, apresentando melhorias práticas no local de trabalho de um ponto de vista ergonômico. Esse manual pode ser utilizado por qualquer pessoa interessada em criar um ambiente de trabalho melhor: empregadores, supervisores, trabalhadores, inspetores, pessoal de segurança e saúde, treinadores e educadores, engenheiros, ergonomistas e designers. [OIT, IEA, 2018]. As informações contidas neste manual foram viabilizadas por meio de um aplicativo com o mesmo nome disponível para Android e iOS.

Um estudo sobre desenvolvimento de aplicativo móvel na prevenção dos fatores de riscos osteomusculares relacionados ao trabalho de enfermagem em unidade hospitalar, apresentou a sua fase de validação do desenvolvimento do aplicativo denominado "DORT", com objetivo de análise da metodologia do desenvolvimento e da usabilidade, realizada por enfermeiros e profissionais de informática. Como resultado, foi recomendada simplificação das funcionalidades do aplicativo para que apresente maior conformidade com a população a que se destina. A versão final do aplicativo será disponibilizada inicialmente para o Hospital Universitário Antônio Pedro, da Universidade Federal Fluminense [Tavares, Gama, 2019]. O aplicativo não foi encontrado nas lojas virtuais.

No estudo preliminar, constando da etapa 1 do desenvolvimento do app Ergon, foram analisados os cinco aplicativos informativos de ergonomia encontrados nas lojas virtuais, e somente um aplicativo, Pontos de Verificação Ergonômica, mais se aproximou das condições de trabalho e itens da NR 17, porém esse app não se relacionou a todos os itens desta norma, além de abordar questões de segurança junto às de ergonomia. A partir dessa análise buscou-se elaborar o aplicativo Ergon baseado na NR 17, possibilitando às empresas e trabalhadores informação para conscientização e implementação atendendo às recomendações da Secretaria do Trabalho do Brasil, que determina através desta norma os parâmetros de ergonomia para adaptação das condições de trabalho ao trabalhador [Brasil, 2018].

\section{Conclusão}

A educação em saúde, por meio da Telessaúde, pode transpor as dificuldades para a realização dos treinamentos de conscientização ergonômica e o distanciamento do trabalhador ao acesso da informação ergonômica. Essas informações podem contribuir com a redução de LER/DORT, além de motivar os trabalhadores inseridos em uma organização de trabalho que estimula uma cultura de cuidados com saúde e segurança. 
Os dispositivos móveis podem contribuir com uma forma flexível de treinamento durante a permanência do trabalhador no seu local de trabalho quando the for possível ou desejável.

O aplicativo Ergon está em desenvolvimento, especificamente com a etapa de análise dos aplicativos informativos em ergonomia, definição do conteúdo e objetivo do aplicativo e a etapa de planejamento das interfaces e da navegabilidade concluídas, e explora o potencial das tecnologias móveis para treinamentos de conscientização em ergonomia para empresas e trabalhadores, em especial àqueles com dificuldade de acesso à essa informação, visando os cuidados para obtenção de um ambiente de trabalho seguro e saudável.

\section{Referências}

Iida, I. and Guimarães, L. B. (2016) "Ergonomia: Projeto e Produção". Editora Edgard Brücher. São Paulo: $3^{\mathrm{a}}$ edição.

ABERGO (2008). "Definição internacional de ergonomia". Revista Ação Ergonômica". Volume 3, Número 2.

Brasil (2018). "LER/DORT afastaram 22 mil trabalhadores das atividades profissionais em 2017". Secretaria do Trabalho. Ministério da Economia. Disponível em: http://trabalho.gov.br/noticias/6194-ler-dort-afastaram-22-mil-trabalhadores-dasatividades-profissionais-em-2017.

Junior, J. C. R (2016). "Gestão e Negócios - Entenda por que as empresas estão migrando para o interior". Conube. Disponível em: https://conube.com.br/blog/entenda-por-que-empresas-estao-migrando-para-ointerior/.

Oliveira, L. B. et al (2015). "Efeitos da Tecnologia Móvel sobre a Qualidade de Vida no Trabalho". Revista Gestão \& Tecnologia, 15(2), pp. 161-185.

Godderis, L and Lambrechsts, M. C. (2019). "Make healthy employees a priority and prevent chronic diseases". International Labour Organization. Available from: https://www.ilo.org/global/topics/safety-and-health-at-work/events-training/eventsmeetings/world-day-for-safety/33thinkpieces/WCMS_681592/lang--en/index.htm

OIT, IEA. (2018) "Pontos de verificação ergonômica: soluções práticas e de fácil aplicação para melhorar a segurança, a saúde e as condições de trabalho". Fundacentro, $2^{\mathrm{a}}$ edição.

Tavares, C. M. M. and Gama, L. N. (2019) "Desenvolvimento e avaliação de aplicativo móvel na prevenção de riscos osteomusculares no trabalho de enfermagem". Texto \& Contexto Enfermagem, 28, e20180214. Epub July 18, 2019. Disponível em: http://www.scielo.br/scielo.php?script=sci_arttext\&pid=S010407072019000100349\&lng=en\&nrm=iso.

Brasil (2018). Normas Regulamentadoras. Disponível em: https://enit.trabalho.gov.br/portal/images/Arquivos_SST/SST_NR/NR-17.pdf. 